\title{
Conformational Characteristics of Free and Cesium Complexed $p$-H-Calix[6]arene Alkyl Ester Derivatives
}

\author{
Kee-Choo Chung, Kyung-Sun Song, Kyoung Nam Kim, Janggeun Cho, Suk-Kyu Chang, and Sangdoo Ahn \\ Department of Chemistry, Chung-Ang University, Seonl 156-756, Korea. "E-mail: sangdoo@ycanac.kr \\ Received Angust 17, 2007
}

Key Words : p-I I-Calix[6]arene, 1,2-Nlternate conformation, Cesium complex, $C_{31}$ conformation, NMR

Calixarenes have received much interest because of their versatile recognition properties toward a variety of ionic and organic guests including biomolecules, which result from the ease of functionalization at the lower rim or upper rim. ${ }^{1-4}$ The conformational characteristics of calixarenes are also known to have strong effects on their complex formation behaviors. ${ }^{1}$ Among the calixarene series, the appropriately functionalized calix[6]arenes are known to have a preference for cesium ion, which is one of the major components of nuclear wastes. ${ }^{1.5}$ Nevertheless, there are only a few reports on the complexation of cesium ion by calix[6]arenes and on the conformational behaviors of the complex and/or the free host itself. ${ }^{67}$ In this paper, we report the unusual conformational characteristics of $p$-H-calix[6]arene derivatives and their cesium ion complexes revealed by using ' $\mathrm{H}$, ${ }^{13} \mathrm{C}$ and ${ }^{133} \mathrm{Cs} \mathrm{NMR}$ spectroscopy at various temperatures.

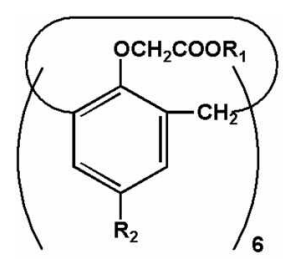

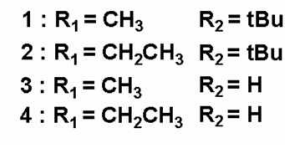

Because of the fast interconversion between conformations, at temperatures above $238 \mathrm{~K}$, the ${ }^{1} \mathrm{H}$ NMR spectra of free $p-\mathrm{H}$-calix[6]arene hosts, 3 and 4, maintained simple patterns. However, below $238 \mathrm{~K}$, the peaks have started to split into multiple peaks, and finally the spectrum changed into a complicated one at $198 \mathrm{~K}$, as shown in Figure 1. The sharp splitting occurred at $198 \mathrm{~K}$ means that the site exchange takes place more rapidly compared to $p$-tert-butylcalix[6]arene host 1 , which showed a fully splitted ${ }^{1} \mathrm{H}$ spectrum at $238 \mathrm{~K} .{ }^{7}$ The $1 \mathrm{D}$ and $2 \mathrm{D}-\mathrm{NMR}$ spectra of the free host 4 at $198 \mathrm{~K}$ are well resolved and allow us to infer the conformational characteristics. The COSY spectrum shows that the phenyl proton resonances are constituted of nine different sites (six for meta and three for para protons), and the possible conformations which give rise to those splitting patterns are 1,2-altemate and/or 1,2,4-altemate forms. The ${ }^{13} \mathrm{C}$ NMR spectrum also supports the above inference, because the carboxyl carbon and the aromatic carbons are divided into three parts with a $1: 1: 1$ intensity ratio, as shown in Figure 1.

Besides the NMR splitting pattems reflecting the molecular symmetry, the absolute positions of ${ }^{13} \mathrm{C}$ signals for the bridged methylenes have been generally used to elucidate calixarene conformations in solution. ${ }^{8-10}$ According to their symmetries, both 1,2-altemate and 1,2,4-altemate conformers may have four different positions (ratio of $1: 2: 2: 1$ ) of the bridged methylene carbons. For the free host 4 , as shown

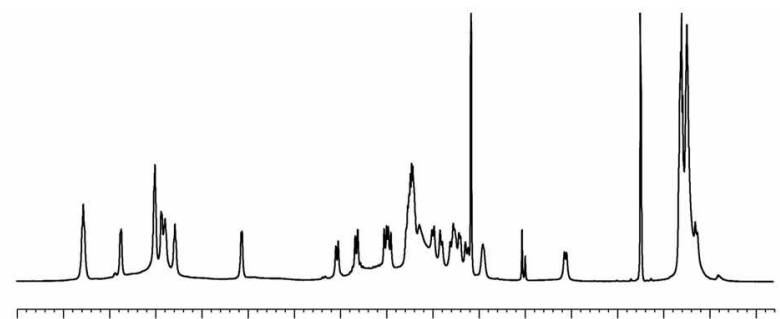

$\begin{array}{lllllllllllllllll}8.0 & 7.5 & 7.0 & 6.5 & 6.0 & 5.5 & 5.0 & 4.5 & 4.0 & 3.5 & 3.0 & 2.5 & 2.0 & 1.5 & \mathrm{ppm}\end{array}$
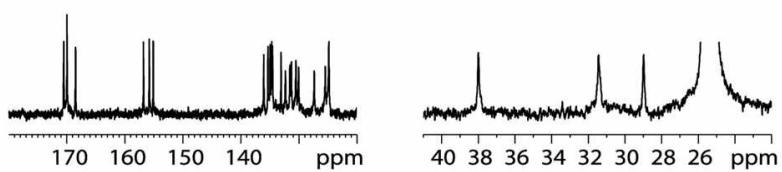

Figure 1. ${ }^{1} \mathrm{H}$ (top) and ${ }^{13} \mathrm{C}$ NMR (bottom) spectra of free host 4 at $198 \mathrm{~K}$ in THF-ds and acetone- $\mathrm{d}_{6}(5: 1, \mathrm{v} / \mathrm{v})$.

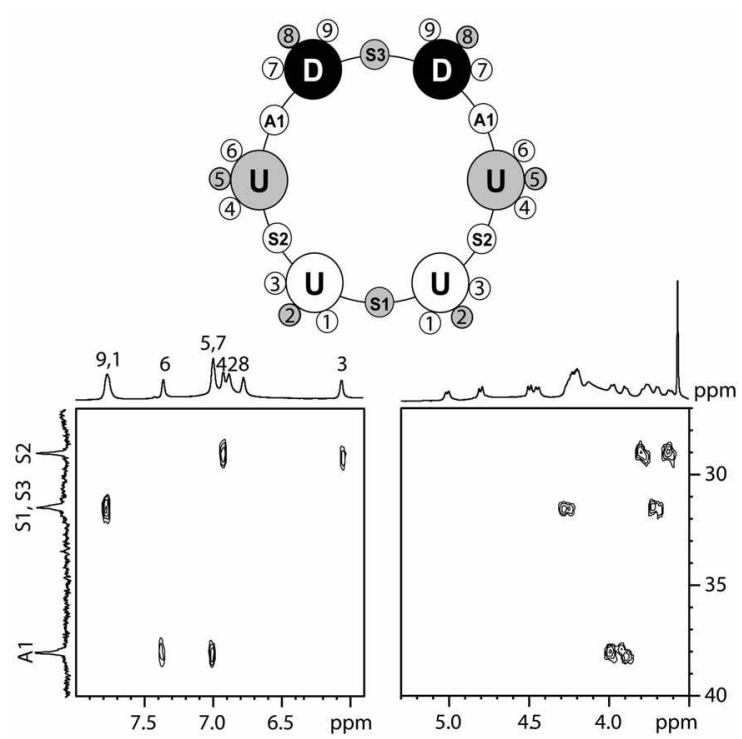

Figure 2. Partial HMBC (left) and HMQC (right) spectra of free host 4 at $198 \mathrm{~K}$ in THF- $\mathrm{d}_{8}$ and acetone-d $(5: 1, \mathrm{v} / \mathrm{v})$, which show the multiple and single bond information between corresponding protons and carbons. Large, medium and sinall circles represent aryl moieties, bridged methylenes and phenyl protons, respectively. 
in Figure 1, only three bridged methylene carbon peaks are observed; one at $38 \mathrm{ppm}$ and two near $31 \mathrm{ppm}$ (29 and 32 $\mathrm{ppm}$ ) which represent anti and syn orientations of connected phenyl groups, respectively. ${ }^{8-10}$ The $\mathrm{C}-\mathrm{H}$ correlation NMR experiments ( $\mathrm{HMBC}$ and $\mathrm{HMQC}$ ) have revealed that the two swn bridged methylene carbon signals ( $\mathrm{S} I$ and $\mathrm{S} 3$, see Figure 2) have been overlaid at $32 \mathrm{ppm}$. These data indicate that $p$-H-calix[6]arene ethyl ester derivative 4 preferentially adopts an unusual 1,2-alternate conformation at low temperature. In contrast, if it were a 1,2,4-altemate conformation, there would be more carbon peaks around $37 \mathrm{ppm}$ than around $31 \mathrm{ppm}$. To our knowledge, there is no report that shows a 1,2-altenate confommation for calix[6]arene derivatives. Also, this behavior is different from host 1 having tertbutyl groups on the upper rim, as we have reported earlier, which has a 1,2,3-altenate conformation at low temperature. Most of simple calix[6]arenes reported have cone or 1,2,3altemate comformers. ${ }^{6-8}$ The methyl ester analogue 3 has shown similar splitting pattens at low temperature, but it was found to have no such preferential conformation at $198 \mathrm{~K}$.

Upon complexation with cesium ion, the methylene resonances ( $\mathrm{ArCH} \mathrm{H}_{2} \mathrm{Ar}$ ) of hosts $\mathbf{3}$ and $\mathbf{4}$ split into a pair of doublets around $3.5-4.5 \mathrm{ppm}$, indicating that the complexes formed have a cone conformation. There are also two broad signals (with a 2:1 intensity ratio) for the aromatic protons in the ${ }^{1} \mathrm{H}$ NMR spectrum. However, as the temperature lowered, the aromatic peaks of $\mathbf{3}$ and $\mathbf{4}$ separated into four peaks corresponding to two sets of 2:1 ratios with the same integral values (see Figure 3). Additionally, the methylene peaks in
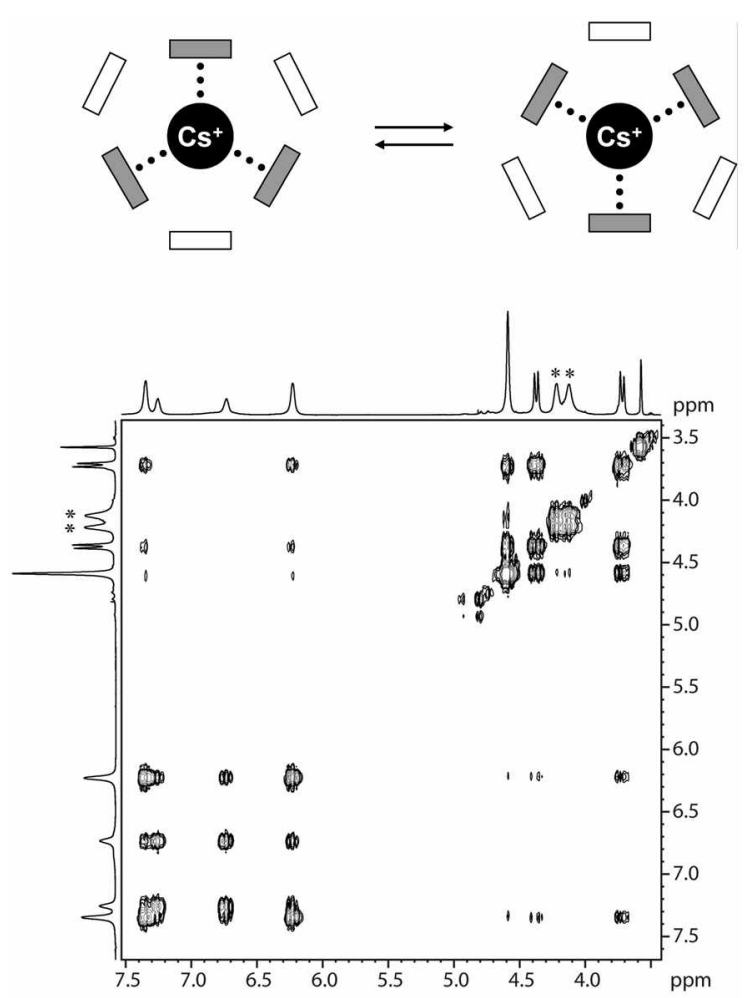

Figure 3. Site exchange scheme (top) and the EXSY spectrum of $4+\mathrm{Cs}^{+}$at $238 \mathrm{~K}$ (bottom) in THF-d and acetone- $\mathrm{d}_{6}(5: \mathrm{l}, \mathrm{v} / \mathrm{v})$. The two methylene peaks in $\mathrm{COOCH}_{2} \mathrm{CH}_{3}$ are marked by asterisks.
$\mathrm{COOCH}_{2} \mathrm{CH}_{3}$ of 4 separated into two peaks (at 4.2 and 4.1 $\mathrm{ppm}$ ) of equal intensity at low temperature. All these observations imply that there are site exchanges between two equivalent conformations that have $C_{3 v}$ symmetry. The existence of this kind of site exchange could be confirmed by the cross peaks between two sets of phenyl proton peaks and between two methylene peaks in $\mathrm{COOCH}_{2} \mathrm{CH}_{3}$ of 4 in the EXSY NMR spectrum at $238 \mathrm{~K}$, as shown in Figure 3. These kinds of conformational ${ }^{6}$ and site exchange ${ }^{11}$ were also observed in the complex $2+\mathrm{Cs}^{+}$.

The exchange rate constant $k$ for the conformational exchange can be obtained by line-shape fitting, and the results for the $3+\mathrm{Cs}^{+}$complex are shown in Figure 4 . The calculations reproduced temperature-dependent NMR spectra successfully for both of the cesium complexes of $\mathbf{3}$ and $\mathbf{4}$. These results support that the site exchange between two equivalent $C_{3 v}$ conformers occurs over the observed range of temperatures.

Although the compound 1 does not complex well with the $\mathrm{Cs}^{+}$ion, weak signals due to the cone-type complex were observed in the 'H NMR spectrum for the mixture of 1 and $\mathrm{Cs}^{t}$. As seen in the other complexes, the signals from the complex have changed at low temperature. For example, the aromatic and tert-butyl proton signals were broadened and finally separated into two peaks, while a pair of doublets from $\mathrm{ArCH}_{2} \mathrm{Ar}$ remained the same throughout the observed temperature range. This is again interpreted as the site exchange between two equivalent conformations having $\mathrm{C}_{3 \mathrm{v}}$ symmetry, which can be seen in other cesium complexes of 2,3 and 4 .

The ${ }^{133} \mathrm{Cs}$ spectra show that all the hosts form endo-type complexes with cesium ions because the cesium resonance peaks undergo upfield shifts referenced to free cesium ion differing from the complexes of calix[4]arene derivatives. ${ }^{12}$ As shown in Figure 5, however, the upfield shifts of the $\mathrm{Cs}^{+}$ complexes of $\mathbf{3}$ and $\mathbf{4}$ are much smaller ( $c a .-8 \mathrm{ppm}$ ) than

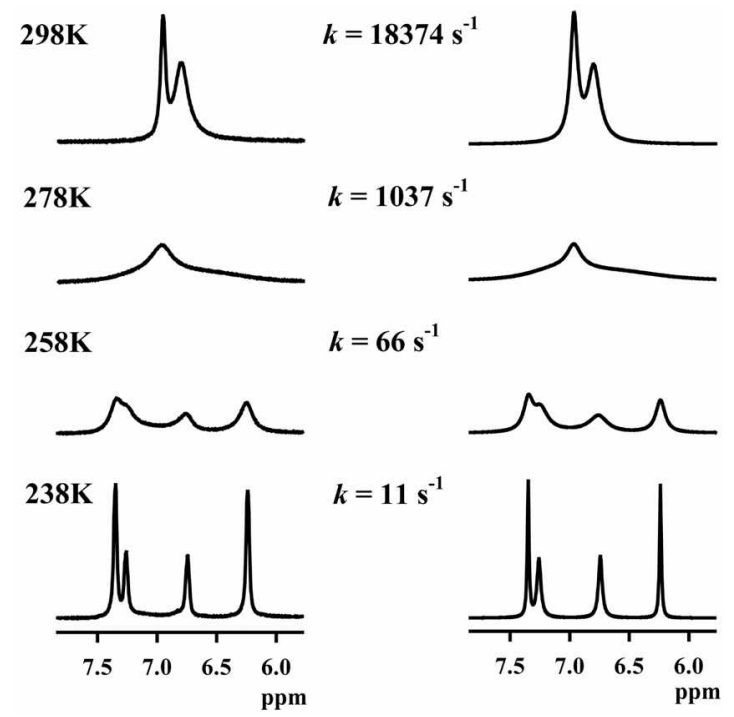

Figure 4. Temperature-dependent 'H NMR line-shapes of phenyl protons in $3+\mathrm{Cs}^{-}$in THF-d $\mathrm{d}_{8}$ and acetone- $\mathrm{d}_{6}(5: 1, \mathrm{v} / \mathrm{v})$. Observed (left) and fitted (right) spectra with indicated exchange rate $k$. 

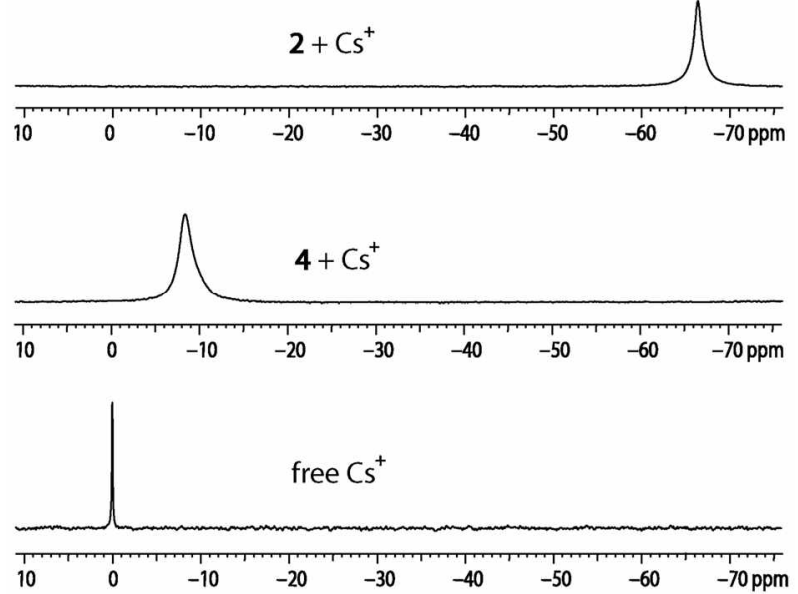

Figure 5. ${ }^{133} \mathrm{Cs}$ spectra of frec $\mathrm{Cs}^{-}, 4+\mathrm{Cs}^{-}$, and $2+\mathrm{Cs}^{-}$at $298 \mathrm{~K}$ in THF $-d_{8}$ and acetone- $d_{6}(5: 1, v / v)$. Note that the chernical shifts are referenced to free cesium ions.

those of 1 and $2(c a-70 \mathrm{ppm})$. This might be caused by the absence of tert-butyl groups on the upper rim of calix[6]arene. The presence of tert-butyl groups makes it easier to lock the cesium ion deeply into the aromatic cavity, where it experiences stronger ring currents.

In summary, we have found that $p$-H-calix[6]arene ethy] ester derivative 4 preferentially adopts an unusual 1,2altemate confonmation at low temperatures. The endo-type complex formation of $\mathbf{3}$ and $\mathbf{4}$ with cesium ions was confirmed by $\mathrm{Cs}^{+} \mathrm{NMR}$, and the complexes were found to undergo mutual interconversions between two equivalent conformations having $C_{3 v}$ symmetry over the observed temperatures.

\section{Experimental Section}

The calix[6]arene derivatives 1-4 were prepared following the reported standard procedures. ${ }^{13}$ Cesium picrate was prepared by the treatment of cesium hydroxide with picric acid and purified by crystallization from ethanol. Due to the limited solubility of calixarenes and cesium picrate (as a guest), a binary mixture of THF- $\mathrm{d}_{8}$ and acetone- $\mathrm{d}_{6}(5: 1, \mathrm{v} / \mathrm{v})$ was used for the NMR experiments. All the deuterated solvents were purchased from Aldrich and Sigma Chemical $\mathrm{Co}$. and used without further purification. ID and 2D-NMR experiments were performed on Bruker Avance 600 and 800 spectrometers. The ${ }^{1} \mathrm{H}$ and ${ }^{13} \mathrm{C}$ NMR spectra were referenced to $\mathrm{THF}$ and the ${ }^{133} \mathrm{Cs}$ NMR spectra were referenced to free cesium ions. The concentration of all solutions was fixed at $0.05 \mathrm{M}$.

Acknowledgements. This work was supported by Korea Research Foundation Grant (KRF-2004-042-C00062) and by Seoul R\&DB program. This study made use of the NMR facility at Korea Basic Science Institute, which is supported by Bio-MR Research Program of the Korean Ministry of Science and Technology (E27070).

\section{References}

I. Gutsche, C. D. Calixarenes Revisited; Royal Society of Chemistry: Cambridge, 1998: 125.

2. Lee, Y. J.; Ko, S. W.; Yeo, H. M.; Jeong. H.; Nam, K. C. Bull. Korean Chent. Soc. 2006, 27, 1227.

3. Akine, S.; Goto, K.; Kawashima, T. Bull. Chem. Soc. Jpn, 2005 , $78,169$.

4. Oshima, T.; Higuchi, H.; Ohto, K.; Inoue, K.; Goto, M. Langmin 2005, 21, 7280 .

5. Beasley, T. M.; Jennings, D. Emiron. Sci. Technol. 1984, $/ 8,207$.

6. Otsuka, H.; Araki, K.; Shinkai, S. Tetrahedron 1995, $51,8757$.

7. Ahn, S.; Chang, S.-K.; Lee, J. W. J. Chem. Soc. Perkin Trans.2 1996, 79 .

8. Kanamathareddy, S.; Gutsche, C. D. J. Ong. Chem. 1994, 59, 3871 .

9. Magrans, J. O.; de Mendoza, J.; Pons, M.; Prados, P. J. Org. Cheni. 1997. 62, 4518.

10. Bifulco, G.; Gomez-Paloma, L.; Riçcio, R.; Gaeta, C.; Troisi, F.; Neri, P. Org. Lett. $2005,7,5757$.

11. Chung, K.-C.; Namgoong, H.; Let, J. W. Bull. Korean Chem. Soc. 2004. 25.616.

12. Meier, U. C.; Detellier, C. J. Chem. Soc. Dalton Trans. 2003, 4574.

13. Arnaud-New, F.; Collins, E. M.; Ferguson, G; Harris, S. J.; Kaitner, B.; Lough, A. J.; McKervey, M. A.; Ruhl, B. L.; Schwing-Weill, M. J.; Seward, E. M. J. Am. Chem. Soc. 1989, J1/,8681. 